\section{Eletromiografia da laringe: estudo da contribuição diagnóstica em 30 pacientes com imobilidade de prega vocal}

Larynx electromyography: study of the diagnostic contribution in 30 patients carrying vocal fold immobillity

\begin{abstract}
Agrício N. Crespo ${ }^{1}$, Aline E. Wolf ${ }^{2}$ Paulo A. Kimaid ${ }^{3}$,
\end{abstract} Elizabeth Quagliato ${ }^{4}$, Maura Viana ${ }^{5}$
Palavras-chave: Eletromiografia, laringe, paralisia. Key words: Eletromiography, larynx, paralysis.

Resumo / Summary

I

ntrodução: A eletromiografia (EMG) é utilizada para diagnóstico e prognóstico de doenças neuromusculares. Objetivo: avaliar a contribuição da EMG no diagnóstico da imobilidade de prega vocal. Forma de estudo: Clínico prospectivo. Material e Método: Para avaliação da contribuição diagnóstica trinta pacientes com imobilidade de prega vocal foram divididos em três grupos de acordo com a hipótese diagnóstica firmada clinicamente e submetidos a exame eletromiográfico de laringe. Resultados: A EMGL diagnosticou lesão neuropática periférica, lesão neuropática central ou fixação cricoarinetóidea em todos os casos de prega vocal imóvel sem causa definida. Nos casos de prega vocal imóvel por trauma mecânico definido clinicamente, a EMGL confirmou lesão neuropática periférica em $70 \%$ dos casos, e determinou outra causa em 30\% (neuropatia por compressão, miopatia e fixação cricoaritenóidea). Nos pacientes com prega vocal imóvel por possível compressão tumoral definida clinicamente, a EMGL confirmou lesão neuropática crônica. Conclusão: A EMGL contribui para a precisão do diagnóstico da imobilidade de prega vocal.

\begin{abstract}
Introdução: Electromyography (EMG) is a technique developed and used in neurology for diagnosis and prognostic definition of neuromuscular diseases. Study design: Clinical prospective. Material and method: Thirty (30) patients with vocal fold immobility have been grouped according to the diagnostic hypothesis clinically established. Results: EMGL diagnosed peripherical neuropathic injury, central neuropathic injury or fixation of the cricoarterytenoideous in all patients who presented vocal fold immobility with no defined cause. In those patients carrying vocal fold immobility on account of mechanical traumatic cause, clinically so defined, EMGL confirmed peripheral neuropathic injury in $70 \%$ of the cases and for the remaining 30\% of the cases, it determined other causes such as peripheral neuropathic of compression, myopia and fixation of the cricoarterytenoideous. Conclusion: EMGL confirmed a chronic neuropathic injury in those patients carrying vocal fold immobility by virtue of compression caused by a possible clinically defined tumor.
\end{abstract}




\section{INTRODUÇÃO}

A eletromiografia (EMG) é técnica amplamente desenvolvida e utilizada em neurologia para diagnóstico e definição prognóstica em diversas doenças neuromusculares. Consiste na captação de potenciais de ação muscular através de eletrodos posicionados na superfície ou inseridos na musculatura. A eletromiografia laríngea (EMGL) é pouco utilizada na prática clínica em nosso meio. De acordo com a literatura, a EMGL traz informações relevantes para o diagnóstico, prognóstico ${ }^{1,2,3,4,5}$ e topodiagnóstico ${ }^{6}$ de alterações da mobilidade laríngea. Contribui, também, para a localização do músculo intrínseco na injeção de toxina botulínica?.

A primeira referência à EMGL encontrada na literatura, de 1944, já define a técnica como recurso complementar à avaliação clínica ${ }^{8}$. Desde então, o estudo da atividade elétrica dos músculos intrínsecos da laringe vem despertando o interesse dos pesquisadores.

A EMGL pode determinar o tipo de doença, contribuir para o topodiagnóstico e diferenciar desordens centrais de periféricas ${ }^{6}$. A avaliação eletromiográfica dos músculos tiroaritenóideo (TA), cricotiróideo (CT) e cricoaritenóideo posterior (CAP) proporciona a diferenciação entre alteração neuropática, miopática, ou decorrente de fixação de cartilagens?.

Traçados eletromiográficos normais indicam anquilose cricoaritenóidea ou luxação. Traçados característicos de desenervação sugerem comprometimento periférico. Padrão eletromiográfico sem desenervação, com traçado rarefeito e potenciais de ação da unidade motora (PAUMs) com morfologia normal, indica comprometimento do sistema nervoso central e, por fim, padrões miopáticos, indicam comprometimento muscular ${ }^{10}$.

A avaliação de alteração neurológica da laringe não deve se restringir apenas à videoendoscopia. É importante que inclua, também, exame clínico, videoestroboscópico e eletromiográfico, tornando-se valiosa na investigação dos mecanismos de controle motor laríngeo, determinando a natureza e o local da lesão e influenciando na decisão terapêutica.

Geralmente, imobilidades de prega vocal sem recuperação espontânea por períodos de observação maior do que seis meses são consideradas irreversíveis e os pacientes são submetidos a tratamento cirúrgico ${ }^{11}$. Nas situações em que os sinais eletromiográficos indiquem prognóstico desfavorável, esse tempo de observação pode ser dispensado.

A EMGL tem sido pouco utilizada em nossa prática clínica. Acreditamos que este fato seja conseqüencia das dificuldades técnicas que se referem a técnica de inserção, tipos de eletrodo e variação anatômica, e dificuldades de interpretação do traçado eletromiográfico pois o otorrinolaringologista é familiarizado com a anatomia e fisiologia laríngea e nem sempre conhece os achados eletrofisiológicos. É um exame que necessita de equipe composta por otorrinolaringologista e eletrofisiologista. Além disso, persistem controvérsias quanto à sua verdadeira eficácia como recurso complementar ao diagnóstico e à conduta terapêutica? .

O tempo decorrido entre o início da imobilidade e a realização do exame eletromiográfico é importante para a interpretação dos achados. Com a realização da EMGL podese obter traçados eletrofisiológicos indicativos de lesões agudas ou crônicas. A EMGL perde valor quando desvinculada dos achados clínicos e da história do paciente.

O objetivo deste estudo é avaliar a contribuição diagnóstica da eletromiografia nas alterações de mobilidade de prega vocal.

\section{MATERIAL E MÉTODO}

Foram avaliados 30 pacientes com imobilidade de prega vocal, atendidos pelo Centro Campinas de Otorrinolaringologia e Cirurgia de Cabeça e Pescoço, de maio de 2000 a maio de 2001. As idades variavam de 18 a 79 anos (média: 53,7), sendo 15 do sexo masculino e 15 do sexo feminino. Os critérios de inclusão na amostra levaram em consideração a presença de imobilidade de prega vocal, com diagnóstico firmado por videolaringoscopia, pelo mesmo otorrinolaringologista, e idade superior a 18 anos.

Para estudo da contribuição da EMGL ao diagnóstico, os pacientes foram separados em três grupos de acordo com a hipótese diagnóstica firmada clinicamente, com base na história pregressa do doente, no exame físico e em outros exames subsidiários: grupo A (sem causa definida - 16 casos), grupo B (imobilidade decorrente de trauma -10 casos, sendo nove de origem cirúrgica e o décimo decorrente de compressão externa) e grupo C (imobilidade decorrente de possível compressão tumoral - quatro casos).

Todos os pacientes foram submetidos a eletromiografia laríngea (EMGL) com exames padronizados, utilizando-se sempre o mesmo equipamento, pelos especialistas da equipe de autores deste trabalho (otorrinolaringologista e eletroneuromiografista).

Foram estudados os músculos tiroaritenóideo (TA) em todos os pacientes, cricotiróideo (CT) e cricoaritenóideo posterior (CAP) quando possível tecnicamente, ou seja naqueles em que os referenciais anatômicos de superfície permitiam a localização precisa do músculo, utilizando-se a seqüência:

- punção do músculo TA à esquerda e à direita, com registro dos potenciais elétricos durante respiração espontânea (repouso - contração mínima) e emissão prolongada do /e/ (contração muscular), em pitch habitual;

- punção do músculo CT à esquerda e à direita, com registro dos potenciais elétricos durante respiração espontânea (repouso - contração mínima) e na emissão prolongada do /i/ em pitch agudo (contração muscular); 
- rotação digital da cartilagem tiróide e punção do músculo CAP, com registros dos potenciais elétricos durante a respiração espontânea (contração mínima), emissão prolongada do /e/ em pitch habitual (repouso) e na inspiração profunda (contração muscular máxima).

Os registros foram obtidos com equipamento Nihon Kohden, modelo Neuropack $\sum$ de 4 canais, razão de modo comum de rejeição maior que $94 \mathrm{Db}$, com filtro de banda passante entre 20 a $5000 \mathrm{~Hz}$, base de tempo de $10 \mathrm{~ms} / \mathrm{cm}$ e sensibilidade variando de 100 a $500 \mu \mathrm{V}$. Foram utilizados eletrodos concêntricos de agulha, Nihon Kohden modelo NM 131, de $30 \mathrm{~mm}$. Todos os registros foram gravados em disquetes.

A análise eletromiográfica foi realizada durante o "repouso - menor atividade obtida" e durante a ativação muscular. Os músculos laríngeos não tem ação agonista e antagonista definida claramente como em outros músculos. Podemos observar tal fato pela ausência de silêncio elétrico durante o repouso muscular, e em função disto, usamos o termo repouso muscular para designar a menor atividade obtida. Na avaliação em "repouso muscular", foram observados e classificados os eventos eletrofisiológico: atividade de inserção (AI) - normal ou aumentada; onda aguda positiva (OAP) - ausente ou presente; fibrilação (FIB) - ausente ou presente; fasciculação (FAS) - ausente ou presente; descarga repetitiva complexa (DRC) - ausente ou presente. Durante a ativação muscular foram observados e classificados os eventos eletrofisiológicos: recrutamento das unidades motoras (REC) - normal, aumentado, diminuído; duração dos potencias de ação da unidade motora (DUR) normal, aumentada, diminuída; potencil polifásico (POL) ausente ou presente; amplitude dos potencias de ação das unidades motoras (AMPL) - normal ou aumentada; padrão de interferência do traçado eletromiográfico obtido - rarefeito (R) ou interferencial (INT).

Os registros eletromiográficos determinaram a classificação das lesões quanto ao tipo (neuropático, miopático) e quanto ao tempo de evolução do processo (recente, crônico):

- neuropática periférica ( $2^{\circ}$ neurônio motor) - presença de atividade espontânea e/ou potencias de ação da unidade motora (PAUMs) com duração e amplitude aumentadas e/ou com traçado de esforço máximo rarefeito;

- neuropática central (1ํㅡㄹourônio motor) - ausência de atividade espontânea e/ou potencias de ação da unidade motora (PAUMs) com morfologia normal e com traçado de esforço máximo rarefeito;

- miopática - PAUMs com duração diminuída, amplitude normal ou diminuída e traçado de esforço máximo interferencial;

- recente - presença de atividade espontânea e PAUMs normais;

- crônico - duração e amplitude aumentadas, podendo ocorrer potenciais polifásicos.

Nos casos de imobilidade unilateral, os resultados eletromiográficos nos músculos contralaterais foram utilizados como parâmetro de comparação intra-sujeito. Nos casos de imobilidade bilateral, foram avaliados ambos os lados.

Esta pesquisa foi analisada e autorizada pela Comissão de Ética da Faculdade de Ciências Médicas da UNICAMP.

\section{RESULTADO}

Dos 30 casos estudados, 17 apresentavam imobilidade de prega vocal esquerda, nove, imobilidade de prega vocal direita e quatro, imobilidade bilateral. Em 27 pacientes os exames eletromiográficos apresentaram alteração no mesmo lado imóvel observado na avaliação laringoscópica. Em três casos, os traçados eram normais. Dos 27 alterados, 24 mostraram lesão neuropática, dois eram compatíveis com o diagnóstico de lesão central e, em um único caso, o resultado eletromiográfico indicou lesão miopática (Figura 1).

No grupo A, dos 16 pacientes com imobilidade de prega vocal sem causa definida, a EMGL determinou 12 casos com lesão no nervo periférico (casos 2, 4, 10, 12, 13, 14, 19, 21, 23, 26, 27 e 30), dois casos com fixação cricoaritenóidea (casos 3 e 5) e em dois casos (casos 11 e 25) indicou lesão no sistema nervoso central (Tabela 1, Gráfico 1).

No grupo B, composto por 10 casos cuja história clínica indicava imobilidade de prega vocal por possível trauma, observamos que a EMGL confirmou a lesão do nervo em sete casos (casos 6, 8, 18, 22, 24, 28 e 29) sugeriu lesão do nervo anterior ao trauma por provável compressão (caso 17), indicou lesão de músculo em um caso (caso 7) e indicou fixação cricoaritenóidea em um (caso 15) (Tabela 2 - Gráfico 2).

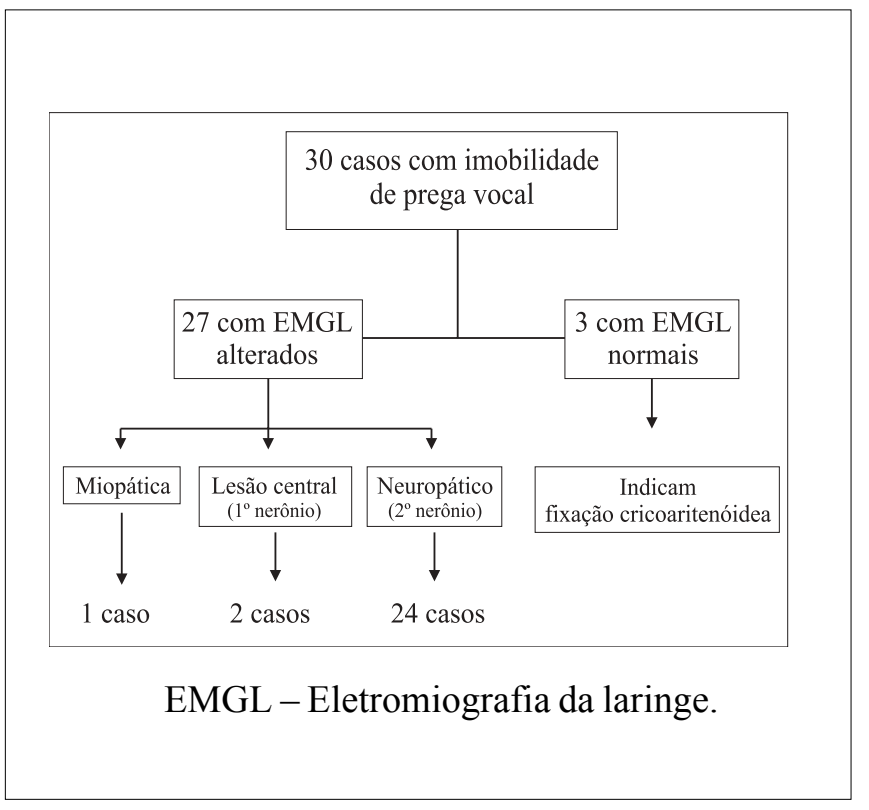

Figura 1. Fluxograma com as interpretações eletromiográficos de 30 casos de imobilidade de prega vocal. 
Tabela 1. Grupo A - Imobilidade de prega vocal sem causa definida $(n=16)$

\begin{tabular}{|c|c|c|c|c|c|}
\hline Caso & Duração & MM & Imob. & InterpretaçõesEMGL & Contribuição da EMGL \\
\hline 2 & $>180 \mathrm{~d}$ & TA E CAP E & IPVE & Lesão neuropática crônica NLR & Define lesão no nervo \\
\hline 4 & $30 \mathrm{~d}$ & TA DCT DCAP D & IPVD & Lesão neuropática recente NLS e NLR, & Define lesão no nervo \\
\hline 10 & $150 \mathrm{~d}$ & TA E/DCAPE/D & IPVB & Lesão neuropática crônica NLR bilateral, & $\begin{array}{l}\text { Define lesão no nervo } \\
\text { bilateral. }\end{array}$ \\
\hline 11 & $150 \mathrm{~d}$ & TA D & IPVD & Diminuição do padrão interferencial & Sugere lesão no SNC \\
\hline 14 & $>180 \mathrm{~d}$ & TA E & IPVE & Lesão neuropática crônica NLR & Define lesão no nervo \\
\hline 19 & $60 \mathrm{~d}$ & TAE & IPVE & Lesão neuropática crônica NLR & Define lesão no nervo. \\
\hline 21 & $90 \mathrm{~d}$ & TA D & IPVD & Lesão neuropáticarecente NLR & Define lesão no nervo \\
\hline 23 & $>180 \mathrm{~d}$ & TA E & IPVE & Lesão neuropática crônica NLR & Define lesão no nervo \\
\hline 25 & $>180 \mathrm{~d}$ & TA E/D & IPVB & Diminuição do padrão interferencial & Sugere lesão no SNC \\
\hline 26 & $>180 \mathrm{~d}$ & TA ECT ECAP E & IPVE & Lesão neuropática crônica NLS e NLR & Define lesão no nervo, \\
\hline
\end{tabular}

Legenda: Imob. - Imobilidade, IPVE - Imobilidade de prega vocal esquerda; IPVD - Imobilidade de prega vocal direita; IPVB - Imobilidade de prega vocal bilateral; NLR - Nervo laringeo recorrente; NLS - Nervo laringeo superior; d - dias; SNC - sistema nervoso central; Duração tempo decorrido do início da queixa até exame eletromiográfico; EMGL - Eletromiografia de laringe. MM. - músculos com alterações, TA músculo tiroaritenoideo, CT- músculo cricoaritenoideo, CAP - músculo cricoaritenoideo posterior, D -direito, E- esquerdo, D/E - esquerdo e direito, $d$ - dias

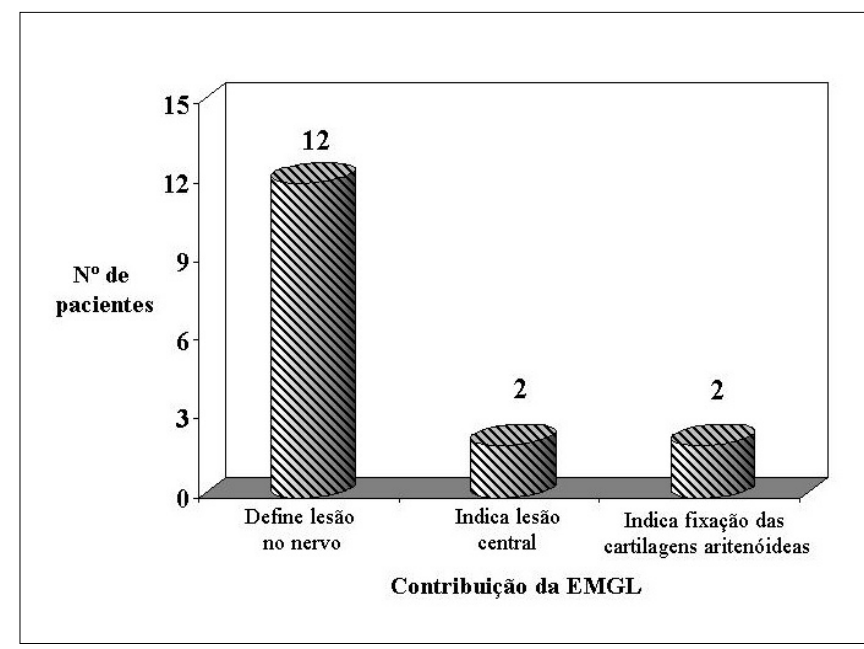

Gráfico 1. Contribuição da EMGL no estabelecimento do diagnóstico no Grupo A - Sem causa definida para a imobilidade da prega vocal. $(n=16)$

No grupo C, composto por quatro casos com hipótese diagnóstica de compressão tumoral, a EMGL revelou indicadores de lesão neuropática de instalação crônica sugerindo um fenômeno compressivo em 100\% dos casos. No caso 20 foi constatada uma discrepância entre o tempo recente de início dos sintomas e os resultados da EMGL, que sugeriram lesão mais antiga (Tabela 3).

\section{DISCUSSÃO}

Desde a descrição original de Weddel e Feinstein ${ }^{8}$ em 1944, muitos trabalhos têm mostrado a importância da EMGL na investigação neurolaringológica, tanto para a conclusão diagnóstica, como para a avaliação de prognóstico ${ }^{1-22}$, embora, em nosso meio poucos estejam familiarizados com a técnica. A avaliação das alterações neurológicas da laringe é complexa e requer exame laringológico, neurológico e estudos eletrofisiológicos que, em muitas situações não se restringe à laringe ${ }^{4,7,19}$.

É consenso que uma prega vocal imóvel possa ser decorrente de alterações no controle central, na inervação, na musculatura ou, ainda, de mal funcionamento da articulação cricoaritenóidea. No entanto, a expressão "paralisia de prega vocal" é utilizada freqüentemente de forma indiscriminada. A laringoscopia e o exame clínico otorrinolaringológico permitem verificar a presença de imobilidade e inferir sobre uma possível causa, lembrando que a freqüência de prega vocal imóvel sem causa definida clinicamente é alta.

A EMGL permite a diferenciação entre alteração na inervação (paralisia), na musculatura (miopatia) e fixação das cartilagens aritenóideas (exame eletromiográfico normal), auxiliando o otorrinolaringologista na determinação diagnóstica e eventualmente modificando sua conduta clínica.

Neste estudo verificamos que no grupo A, constituído por 16 pacientes sem causa definida clinicamente, a EMGL 
Tabela 2. Grupo B - Imobilidade de prega vocal por possível trauma mecânico $(n=10)$

\begin{tabular}{|c|c|c|c|c|c|}
\hline Caso & Duração & MM & Imob. & Interpretação EMGL & Contribuição da EMGL \\
\hline 6 & $>180 \mathrm{~d}$ & TA E & IPVE & Lesão neuropática crônica NLR & Confirma lesão do nervo \\
\hline 7 & $41 \mathrm{~d}$ & TA E & IPVE & Lesão miopática TA D & $\begin{array}{l}\text { Não há lesão do nervo, indica } \\
\text { alteração inflamatória }\end{array}$ \\
\hline 8 & $>180 \mathrm{~d}$ & TA ECT E & IPVE & Lesão neuropática crônica NLS e NLR & Confirma lesão do nervo \\
\hline 15 & $>180 \mathrm{~d}$ & 0 & IPVB & Exame normal & Indica fixação das aritenóides \\
\hline 17 & $120 \mathrm{~d}$ & TA ECT E & IPVE & Lesão neuropática crônica NLR e NLS. & $\begin{array}{l}\text { Lesão no nervo crônica, } \\
\text { possivelmente anterior à } \\
\text { cirurgia. }\end{array}$ \\
\hline 18 & $60 \mathrm{~d}$ & TA D & IPVD & Lesão neuropáticarecente NLR & Confirma lesão do nervo \\
\hline 22 & $60 \mathrm{~d}$ & TA ECT ECAP E & IPVD & Lesão neuropática recente NLR e NLS & Confirma lesão do nervo \\
\hline 24 & $>180 \mathrm{~d}$ & TA DCT DCAPD & IPVD & Lesão neuropática crônica NLR e NLS & Confirma lesão do nervo \\
\hline 28 & $>180 \mathrm{~d}$ & TA ECT ECAP E & IPVE & Lesão neuropática crônica NLR & Confirma lesão do nervo \\
\hline 29 & $>180 \mathrm{~d}$ & TA E & IPVE & Lesão neuropática crônica NLR & Confirma lesão do nervo \\
\hline
\end{tabular}

Legenda: Imob. - Imobilidade, IPVE - Imobilidade de prega vocal esquerda; IPVD - Imobilidade de prega vocal direita; IPVB - Imobilidade de prega vocal bilateral; NLR - Nervo laringeo recorrente; NLS - Nervo laringeo superior;d - dias; SNC - sistema nervoso central; Duração tempo decorrido do início da queixa até exame eletromiográfico; EMGL - Eletromiografia de laringe. MM. - músculos com alterações, TA músculo tiroaritenoideo, CT- músculo cricoaritenoideo, CAP - músculo cricoaritenoideo posterior, D -direito, E- esquerdo, D/E - esquerdo e direito, $\mathrm{d}$ - dias..

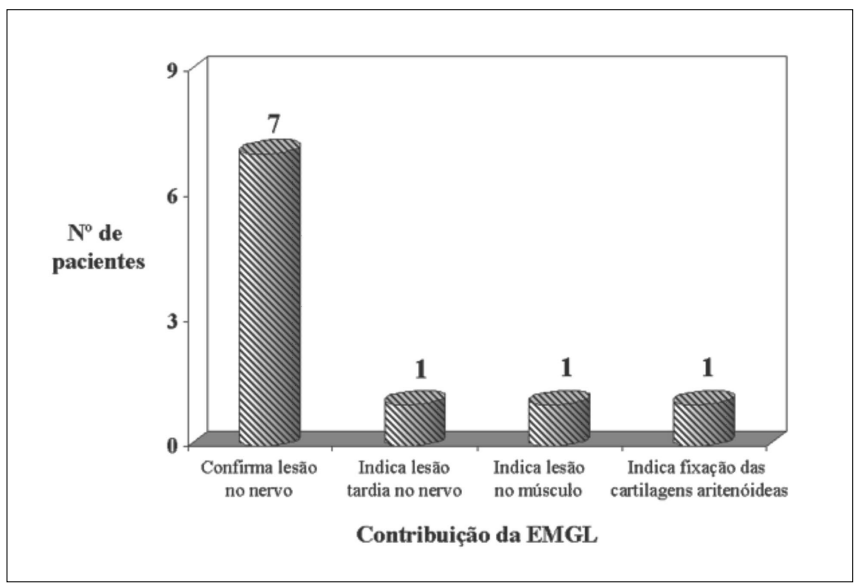

Gráfico 2. Contribuição da EMGL no estabelecimento do diagnóstico no Grupo B - Imobilidade decorrente de trauma definido por história clínica e exames subsidiários $(n=10)$

determinou 12 alterações neuropáticas, duas alterações no sistema nervoso central e dois exames normais sugerindo fixação das cartilagens aritenóideas.

No grupo B, dos 10 pacientes com imobilidade de prega vocal por possível trauma, definido por história clínica e exames subsidiários, oito apresentaram alterações eletromiográficas indicativas de neuropatia, um apresentou exame eletromiográfico normal (caso 15) e um apresentou alterações eletromiográficas indicativas de miopatia (caso 7).

O caso 15 foi inserido no grupo B por apresentar prega vocal imóvel bilateral e alterações vocais com início imediatamente após traumatismo causado por tentativa de enforcamento. A EMGL revelou exame normal, sugestivo de fixação das cartilagens.

O caso 7 apresentou imobilidade de prega vocal esquerda e alteração vocal após tiroidectomia. O resultado eletromiográfico definiu lesão miopatia (potenciais polifásicos de curta duração), tendo sido atribuído ao traumatismo local das fibras musculares devido à entubação orotraqueal, como anteriormente referido na literatura ${ }^{19}$.

No grupo C, composto por quatro casos com imobilidade por possível compressão tumoral, a EMGL corroborou o diagnóstico clínico em todos.

$\mathrm{Na}$ maioria dos casos, os resultados eram de lesões neuropáticas, com características compatíveis com o tempo decorrido entre o início dos sintomas e a realização da EMGL, ou seja, a eletromiografia detectou lesões crônicas em pacientes cuja história era antiga. Entretanto, em dois casos (caso 17 e 20) os resultados eletromiográficos foram discrepantes em relação ao tempo de início dos sintomas informado pelos pacientes, sugerindo se tratar de processo mais antigo.

No caso 20, a causa da imobilidade era compressão tumoral (tumor no timo - diagnosticado por tomografia computadorizada), e os resultados EMGL eram compatíveis com lesão crônica, em discordância como o tempo de instalação da queixa de disfonia, que era recente (34 dias).

No caso 17, o paciente foi submetido a tiroidectomia e segundo relatado, apresentou alteração vocal imediata. A EMGL revelou lesão crônica dos nervos laríngeo recorrente e superior, sugerindo se tratar de lesão anterior à cirurgia. Esta condição ilustra a possível aplicação do método em causas judiciais.

As características eletromiográficas que foram 
Tabela 3. Grupo C - Imobilidade de prega vocal por possível compressão tumoral. ( $n=4)$

\begin{tabular}{ccclll}
\hline Caso & Duração & MM & Imob. & InterpretaçãoEMGL & Contribuição EMGL \\
\hline 1 & $>180 \mathrm{~d}$ & TA E & IPVE & Lesão neuropática crônica NLR & Confirma lesão do nervo \\
9 & $180 \mathrm{~d}$ & TA DCT DCAP D & IPVD & Lesão neuropática crônica NLR e NLS & Confirma lesão do nervo \\
16 & $>180 \mathrm{~d}$ & TA ECT E & IPVE & Lesão neuropática crônica NLR e NLS & Confirma lesão do nervo \\
\hline 20 & $34 \mathrm{~d}$ & TA E & IPVE & Lesão neuropática crônica NLR & $\begin{array}{l}\text { Confirma lesão do nervo, } \\
\text { questiona tempo de história }\end{array}$ \\
\hline
\end{tabular}

Legenda: Imob. - Imobilidade, IPVE - Imobilidade de prega vocal esquerda; IPVD - Imobilidade de prega vocal direita; NLR - Nervo laringeo recorrente; NLS - Nervo laringeo superior; d - dias; Duração - tempo decorrido do início da queixa até exame eletromiográfico; EMGL Eletromiografia de laringe. MM. - músculoscom alterações, TA - músculo tiroaritenoideo, CT- músculo cricoaritenoideo, CAP - músculo cricoaritenoideo posterior, D -direito, E- esquerdo.

discrepantes em relação ao tempo de história nos dois casos acima descritos foram semelhantes às encontradas nos demais pacientes, cuja etiologia era compressão tumoral. Embora poucos casos tenham sido avaliados precocemente, as alterações eletrofisiológicas nos casos traumáticos, onde a instalação da lesão foi abrupta, se mostraram consistentes com o tempo de início das queixas clínicas. Isso nos levou a concluir que, nos casos de paralisia por compressão tumoral, a manifestação clínica pode aparecer muito depois do início das alterações eletrofisiológicas. Provavelmente, esse fato se deva ao comprometimento lento e gradual dos axônios, permitindo o brotamento de prolongamentos colaterais da junção mioneural e a compensação clínica que os demais músculos da laringe, por aumento da ação sinérgica ou redução da antagonista, poderiam propiciar no início do processo.

Nos casos em que a instalação do processo neuropático ocorreu subitamente, a repercussão clínica é evidente e sempre referida pelo paciente, não possibilitando o mecanismo adaptativo proposto acima.

A definição prognóstica depende da comparação com padrões eletrofisiológicos esperados nas diversas fases evolutivas de uma paralisia, a exemplo do que ocorre com o nervo facial. Vários autores estudaram prognóstico experimentalmente na laringe de animais ${ }^{14,15,18}$, e na laringe em humanos ${ }^{1,2,12,23}$. No entanto, estes estudos não apresentaram resultados concordantes. Que critérios prevêem recuperação clínica espontânea da prega vocal imóvel? Quais critérios indicam evolução clínica desfavorável? O restabelecimento da mobilidade da prega vocal é sempre acompanhado de retorno à normalidade eletrofisiológica? Sinais eletromiográficos indicativos de recuperação correspondem à melhora clínica da função laríngea? São perguntas que permanecem com respostas controversas.

O estudo prognóstico das imobilidades da laringe depende de resultados eletromiográficos determinados cronologicamente em intervalos representativos da seqüência de degeneração e regeneração nervosa, já conhecidos em outros nervos ${ }^{24-27}$.
Dos 30 pacientes estudados, 24 realizaram a EMGL após 90 dias do início dos sintomas. A distribuição de nossa amostra (segunda coluna das tabelas 1, 2, 3) revelou que a avaliação neurolaringológica é quase sempre tardia em nosso meio. Interpretamos este fato em função das manifestações vocais encontradas nas imobilidades laríngeas, principalmente nas unilaterais, que, devido a características de tolerabilidade e aceitação social levam o doente a protelar a busca de atendimento médico especializado. Além disso, quando os recursos médicos são solicitados, nem sempre as equipes dominam completamente as possibilidades de contribuição que o exame eletromiográfico pode oferecer pois não está familiarizado com a avaliação neurolaringológica padronizada e com a utilização da EMGL como importante instrumento diagnóstico.

\section{CONCLUSÕES}

Quanto à contribuição diagnóstica do exame eletromiográfico em 30 casos de pacientes com imobilidade de prega vocal, pudemos concluir que:

- A EMGL diagnosticou lesão neuropática periférica, lesão neuropática central ou fixação cricoarinetóidea em todos os casos de prega vocal imóvel sem causa definida.

- Nos casos de prega vocal imóvel por trauma mecânico definido clinicamente, a EMGL confirmou lesão neuropática periférica em $70 \%$ dos casos, indicou lesão neuropática periférica de instalação lenta, sugerindo compressão em $10 \%$ e determinou outra causa em 20\% (miopatia e fixação cricoaritenóidea).

- Nos pacientes com prega vocal imóvel por possível compressão tumoral definida clinicamente, a EMGL confirmou lesão neuropática de instalação crônica.

- A EMGL é importante recurso diagnóstico e depende de correlação clínica.

- Paciente com imobilidade de prega vocal não procura avaliação médica especializada no início dos sintomas e em nosso meio, a EMGL não é realizada nas fases iniciais da lesão. 


\section{REFERÊNCIAS BIBLIOGRÁFICAS}

1. Gupta SR, Bastian RW. Use of laryngeal electromyography in predictio of recovery after vocal cord paralisys. Muscle \& Nerve 1993 Set;977-978.

2. Hirano M, Nozoe I, Shin T, Maeyama T. Electromyographic findings in recurrent laryngeal nerve paralysis. A study of 130 cases. Practice Otologia Kyoto 1974; 67:231-242.

3. Kotby MN, Fadly E, Madkour O, Barakah A, Khidr A, Alloush T, Saleh M. Electromyography in neurolaringology. Journal of Voice 1992;(2)159-187.

4. Lovelace RE, Blitzer A, Ludlow CL. Clinical Laryngeal Electromyography. In: Blitzer A, Sasaki CT, Fahn S, Brin A, Harris KS. - Neurologic Disorders of the Larynx. New York: Thieme Publishers; 1992. p.66-81.

5.Munin MC, Murry T, Rosen CA. Laryngeal electromyography. Diagnosis and prognostic applications. Otolaryngologic Clinics of North America 2000;4:759-771.

6. Rontal E, Rontal M, Silverman B, Kileny PR. The clinical differentiation between vocal cord paralysis and vocal cord fixation using electromyography. Laryngoscope 1993; 103:133-137.

7. Woodson GF. Clinical value of laryngeal EMG is dependent on experience of the clinician. Arch Otolaryngol Head Neck Surg 1998;124(4):476.

8. Weddel GB; Feinstein B. The electrical activity of the voluntary muscles in man under normal and pathological conditions. Brain 1944;67:178-242.

9. Yin SS, Qiu WW, Stucker FJ. Major patterns of laryngeal electromyography and their clinical application. Laryngoscope 1997;107:126-136.

10. Traissac L, Gioux M, Rovira HP, Henry C, Bertrand B. L'Electromyographie (EMG) du larynx dans le diagnostic des immobilités larynges spontanées ou post-thyrö̈dectomie. Revue de Laryngologie 1991;112(3):205-207.

11. Tucker HM. Vocal Cord Paralisis-1979: Etiology and Management. Laryngoscope 1980;89:504-507.

12. Faaborg-Andersen K. Electromyographic investigation of intrinsic laryngeal muscle in humans. Acta Physiol Scand (suppl) 1957;41, suppl 140.

13. Koufman JA, Walker FO, Johardji GM. The cricothyroid muscle does not influence vocal fold position in laryngeal paralysis. Laryngoscope 1995;105:368-372.

14. Mu L, Yang S. Electromyographic study on end-to-end anastomosis of the recurrent laryngeal nerve in dogs. Laryngoscope 1990;100:1009-1017.
15. Mu L, Yang S. An experimental study on the laryngeal electromyography and visual observations in varying types of surgical injuries to the unilateral recurrent laryngeal nerve in the neck. Laryngoscope 1991;101:699-708.

16. Schaefer SD. Laryngeal electromyography. Otolaryngologic Clinics of North America 1991;5:1053-1057.

17. Schweizer V, Woodson GE, Bertorini TE. Single-fiber electromyography of the laryngeal muscle. Muscle\&Nerve 1999;22:111-114.

18. Shindo ML, Herzon GD Hanson DG, Cain DJ, Sahgal V. Effects of denervation on Laryngeal muscle: A Canine Model. Laryngoscope 1992;102:663-669.

19. Simpson DM, Sternman D, Graves-Wrigth J, Sanders I. Vocal Cord paralysis: clinical and electrophysiologic features. Muscle\&Nerve 1993;16:952-957.

20. Verhulst J, Gioux M, Castro E, Quintero R, Traissac L. Intérêt et role de l'electromyographie dans l'evaluation d'um trouble de la mobilité laryngée et son pronostic. Ver Laryngol Otol Rhinol 1995;116(4):289-292.

21. Yin SS, Qiu WW, Stucker FJ. Value of electromyography in differential diagnosis of larngeal joint injuries after intubation. Ann Otol Rhinol Laryngol 1996;105:446-451.

22. Yin S, Stucker F, Qiu WW, Batchelor BM. Clinical Evaluation of Neurolaryngological Disorders - Ann Otol Rhinol Laryngol 109:832-838, 2000.

23. Kimaid PAT, Resende LAL, Quagliato EMAB, Viana MA, Crespo NA, Wolf A. Eletromiografia Laríngea: Estudo normativo do potencial de unidade motora dos músculos tireoaritenoideus e cricotiroideus. Arq Neuropsiquiatr 2001;59(3B):58-59.

24. Desmedt JE, Borenstein S. Collateral innervation of muscle fibres by motor axons of dystrophic motor units. Nature 1973;(246):500501.

25. Dumitru D. Electrodiagnostic medicine. 1 ed. Hanley-Belfus Inc.; 1995:229-234

26. Gorio A, Carmignoto G, Finesso M, Polato P, Nunzi MG. Muscle reinnervation II. Sprouting, synapse formation and repression. Neuroscience 1983;8:403-416

27. Gorio A, Marini P, Zanoni R. Muscle reinnervation III. Motoneuron sprouting capacity, enhancement by exogenous gangliosides. Neuroscience 1983;8:417-429.

28. Kimura J. Electrodiagnosis in Diseases of Nerve and Muscle. 2ed. Philadelphia: F.A. Davis Company; 1989. p.249-274.

29. Wolf AE, Crespo AN, Qualiato E, Kimaid PA, Viana M. Eletromiografia

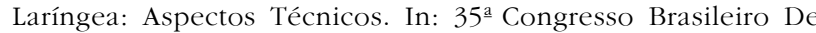
Otorrinolaringologia, Rio Grande do Norte, 2000. Temas Livres, Rio Grande do Norte, Sociedade Brasileira de Otorrinolaringologia, 2000. p. 95.(Resumo, TL-L-12) 